\title{
On the performance of GAIA on photometry of eclipsing binaries: The case of four near-contact and contact systems
}

\author{
P. G. Niarchos and V. N. Manimanis
}

\begin{abstract}
Department of Astrophysics, Astronomy and Mechanics, National \& Kapodistrian University of Athens, GR 15784 Zographou, Athens, Greece

Received 6 March 2003 / Accepted 14 April 2003

Abstract. The light curves of four eclipsing binaries (two near-contact and two contact) are analysed by means of light curve synthesis techniques to derive the geometric and photometric elements and the physical parameters of the systems. For the analysis we used ground based photometric observations and Hipparcos/Tycho photometric data, which mimic the photometric observations that should be obtained by GAIA, the approved Cornerstone 6 mission by ESA. The results are compared and the achievable precision of the basic stellar parameters derived by GAIA photometry is discussed.
\end{abstract}

Key words. surveys - stars: fundamental parameters - stars: binaries: eclipsing

\section{Introduction}

Stellar astrophysics is a cornerstone of fundamental cosmogonic enquiry. In order to approach the broader basic questions properly, we must know, as confidently as possible, the physics of stars and the limits of this knowledge. GAIA is designed to obtain extremely precise astrometry (in the microarcsec regime), multi-band photometry and high resolution spectroscopy for a large sample of stars. This large amount of data of extreme accuracy will stimulate a revolution in the exploration of stellar and Galactic formation and evolution, and the determination of the cosmic distance scale.

The main scientific objectives of the GAIA mission and the expected benefits for astrophysical research are given by Gilmore et al. (1998) and Perryman et al. (2001), an overview of the GAIA payload and spacecraft is presented by Pace (2003), while the goals of GAIA spectroscopy and photometry are discussed by Munari (1999a,b; 2002) and Hoeg (2002). The role of GAIA photometry and spectroscopy on eclipsing binaries has been reviewed in details by Munari et al. (2001) and Zwitter (2002, 2003). The GAIA large-scale photometric survey will have significant intrinsic scientific value for the study of variable stars of nearly all types, including detached eclipsing binaries, near-contact or contact binaries and pulsating stars. It is expected that about $1 \times 10^{6}$ eclipsing binaries (EBs), (with $V \leq 16 \mathrm{mag}$ ), will be discovered. In the fiveyear mission lifetime the number of photometric observations per target and for each one of the 5 broad bands will be about 100 and about 150 for each of the 10 intermediate bands, and the observing fashion will be quite similar to Hipparcos/Tycho mission operational mode. Even if reliable physical parameters could be derived for only $1 \%$ of the observed EBs, this would

Send offprint requests to: P. Niarchos, e-mail: pniarcho@cc.uoa.gr be a great contribution to stellar astrophysics and a giant leap in comparison with what has been obtained so far from ground based observations (Andersen 1991). The study of EB systems provides insights into nearly all areas of astrophysics, including stellar interiors and atmospheres, stellar evolution, nucleosynthesis, plasma physics, magnetic dynamos (in cool stars), and relativistic physics to name a partial list. Recently, the study of EBs in other galaxies and clusters makes it possible to explore stellar evolution and establish mass-luminosity laws for galaxies with vastly different evolutionary and chemical histories from our Galaxy (such as LMC and SMC). Moreover, EBs are beginning to play an important role in cosmology as distant indicators to nearby galaxies. As more data are accumulated, the studies of these systems may lead to an improvement in the extragalactic distance scale.

The aim of the present paper is a first evaluation of the GAIA performance on the photometry of contact and nearcontact EBs by analysing the light curves of selected systems using ground based and Hipparcos photometric observations. The solutions based on Hipparcos observations are presented for the first time and compared with the existing solutions based on ground-based observations. It should be noticed that the same light curve synthesis code (Wilson-Devinney code) and the same fixed parameteres (e.g. spectroscopic mass ratio, effective temperature of the primary component, limb and gravity darkening) were used to secure homogeneity in the light curve solution. It should also be made clear that the spectroscopic data used were not obtained over the GAIA wavelength range with ground-based telescopes to mimic GAIA's own spectral observations, but were taken from the literature and are based on observations in other wavelengths (optical). The ultimate goal of this study is to compare the (absolute) physical parameters of two semi-detached and two contact systems 
derived from ground-based and Hipparcos observations. From these results it is possible to evaluate what could be the impact of GAIA on the research on these stars, taking into account that similar data will be obtained not in one but in 15 diagnostic photometric bands of GAIA.

\section{Selection of systems}

There is an intrinsic limit on the number of photometric observations to the GAIA operational mode ( $\sim 100$ to 150 points per each target). The number of photometric points per band will be similar to that of Hipparcos. However Hipparcos observed in just three bands $H_{P}, B_{T}, V_{T}$, (see Fig. 1 of Munari et al. 2001), while GAIA is baselined to operate 15 bands. Therefore, the total number of photometric points per star expected by GAIA will be $\sim 5$ times more the data provided by Hipparcos. How these observations can be compared with the state-of-the-art ground based observations? What is the accuracy to which EBs can be investigated using GAIA data alone? The aim of the present investigation is to give an answer to the questions above regarding the semi-detached and contact binaries. Such an investigation for detached binaries has been given by Munari et al. (2001), where spectral data over the GAIA wavelength range with ground-based telescopes were used.

In order to answer the above questions we have chosen in our investigation four eclipsing systems, two near-contact (semi-detached) and two contact systems. We have used the following three criteria for the selection of the systems: (i) The systems have been observed by the Hipparcos/Tycho mission. (ii) Ground based photometric observations of high quality exist for the same systems. (iii) Accurate spectroscopic mass ratios (determined from radial velocity measurements using modern techniques) are available.

The systems selected according to the above criteria are the near-contact systems RZ Dra and V1010 Oph, and the contact (W UMa type) systems V566 Oph and $\epsilon$ CrA. The $V$ light curves from ground-based observations and those obtained from Hipparcos observations (filter $H_{P}$ ) are analyzed and the derived elements are compared. Since GAIA photometry will have excellent precision (and in a much larger number of photometric bands), such a comparison has sense, if the most accurate observations of Hipparcos/Tycho mission are used. These are the observations made in the $H_{P}$ band that resemble the white light measurements. The four systems were selected from the Hipparcos sample of bright stars, which is currently the most complete as far as the stellar variability is concerned, down to the amplitude levels of perhaps 0.02 to $0.03 \mathrm{mag}$, and certainly to amplitudes larger than $0.05 \mathrm{mag}$. The light curves obtained by Hipparcos mission in the $H_{P}$ band photometry were retrieved from the HIP database.

\subsection{Remarks to the systems selected}

\section{RZ Dra}

RZ Dra (PPM 36577) has been frequently observed photometrically since its discovery by Ceraski (1907). The most recent extensive analysis of the system was made by
Kreiner et al. (1994). The analysis of their own $B$ and $V$ light curves indicates that the system is a near-contact one, with the secondary, less massive and less luminous component filling its Roche lobe, while the primary is slightly below. A single-lined spectroscopic orbit was derived by Struve (1946) but, very recently, Rucinski et al. (2000) were able to detect the secondary component and measure $K_{2}$. They found a spectral type A6 V (which applies to the primary and hotter component) and a spectroscopic mass ratio $q_{\mathrm{sp}}=0.40 \pm 0.04$ which is slightly different from the photometric one, $q_{\mathrm{ph}} \simeq 0.45$, derived by Kreiner et al. (1994). According to the spectroscopic study by Rucinski et al. (2000), RZ Dra appears to be a relatively short-period (0.55 days) semi-detached Algol-type binary with both components accessible to spectroscopic observations. In the present analysis the $V$ light curve by Kreiner et al. (1994) and the spectroscopic mass-ratio by Rucinski et al. (2000) have been used. The spectroscopic observations were made with a grating giving a dispersion of $0.62 \AA \mathrm{pixel}^{-1}$ in the range 3850-4450 A.

\section{V1010 Oph}

V 1010 Oph (HD 151676, BD-15 $\left.{ }^{\circ} 4395\right)$ is one of the brightest and best studied of the near-contact $(P=0.6614)$ binaries. Since its discovery by Strohmeier et al. (1964) it has been observed photometrically by several investigators (for a history of the system see Corcoran et al. 1991). The light curves show unequal minima and out-of-eclipse light variations typical for stars of this class. The binary is single-lined and typically shows an A-type spectrum in the visible (Popper 1966; Cowley et al. 1969). Shaw et al. (1990) succeeded to determine the spectroscopic mass ratio of the system using IUE observations. This was accomplished by combining radial velocities for the F component from the IUE long wavelength, high dispersion spectra, with ground based radial velocities for the A7 component. According to Shaw (1990), V1010 Oph is the prototype of a subclass of near contact binaries. The period of the binary seems to be decreasing (Lipari \& Sistero 1987), which can be explained in terms of conservative mass transfer (Shaw 1990). The $V$ light curve by Leung (1974) as solved by Manimanis (2003) and the spectroscopic mass ratio found by Shaw et al. (1990) have been used in the present analysis.

\section{V566 Oph}

V566 Oph (HD 163611, BD $+5^{\circ} 3547$ ) is a short period $(P=0.4096)$ A-type W UMa system in which the primary minimum corresponds to a transit eclipse of the larger, more massive and hotter component. After its discovery (Hoffmeister 1935), the system has been the subject of numerous photometric investigations (for a history of V 566 Oph see Niarchos et al. 1993). The light curves of the system had exhibited remarkable constancy in the past (e.g. Boookmyer 1976; Eaton 1986) till Lafta \& Grainger (1985) reported significant changes in the depths of its eclipses. Spectroscopic observations of the system were made by Heard (1965), McLean (1983) and by Hill et al. (1989). The binary is a 

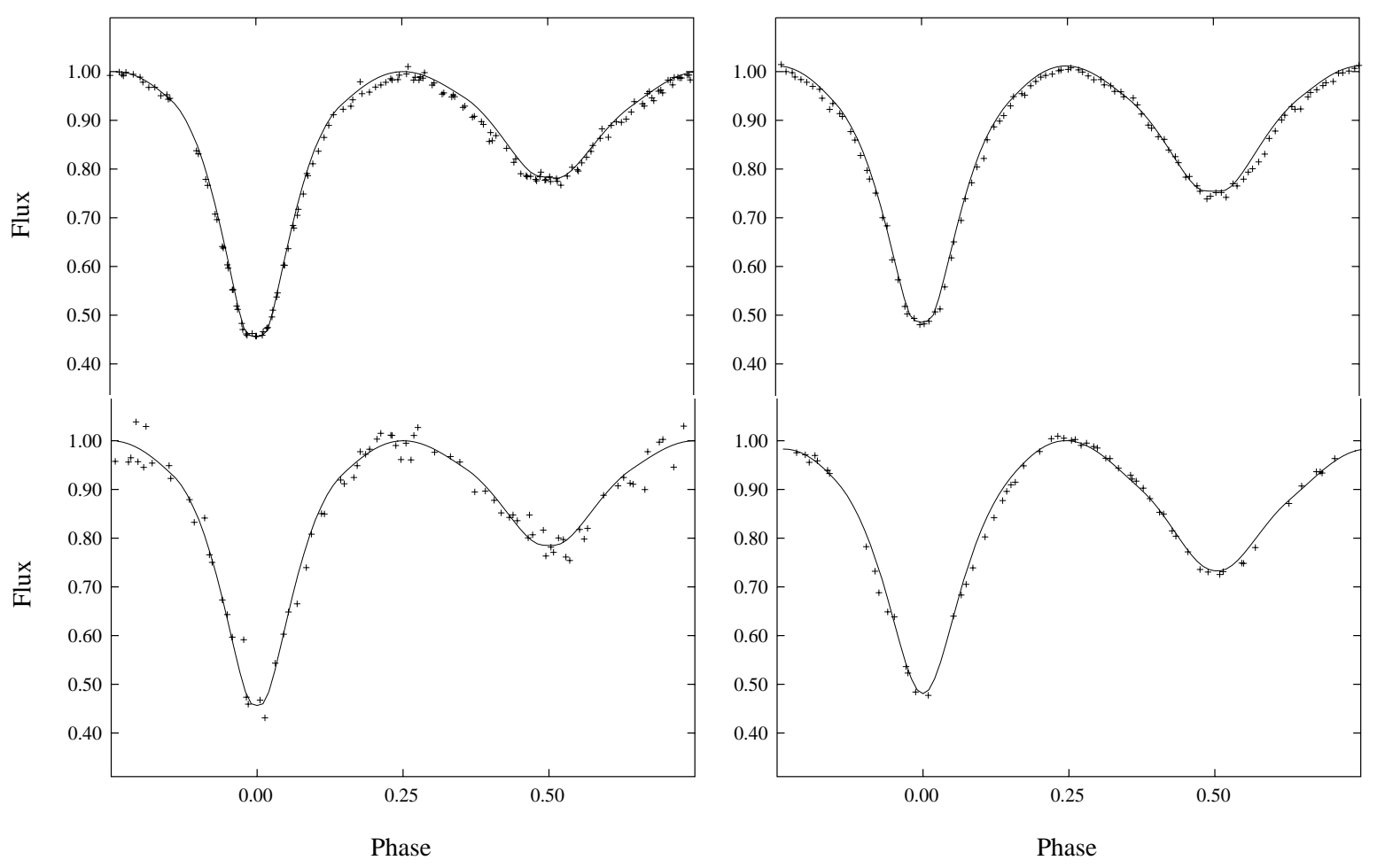

Fig. 1. Light curves of the semi-detached systems RZ Dra (left) and V1010 Oph (right). The upper curves are from ground based observations and light curve solutions (Kreiner et al. 1994 and Leung 1974, respectively). The lower curves are from Hipparcos data and our respective light curve solutions (see text for details).

double-lined system with complete eclipses, so that one should be able to find both spectroscopic and photometric mass ratio reliably. In our analysis we used the $V$ light curve by Bookmyer (1976) as solved by van Hamme \& Wilson (1985) and the velocity curves by McLean (1983). The latter were measured from spectra which had a dispersion of $20 \AA \mathrm{mm}^{-1}$ and were obtained on baked IIa-O plates with an average exposure time of about $25 \mathrm{~min}$.

\section{$\epsilon$ Cra}

$\epsilon$ Cra (HD 175813, DM-37 13001, SAO 210781) is an Atype W UMa system discovered by Cousins \& Cox (1950). With an apparent magnitude of $4.73, \epsilon$ Cra is the apparently brightest known W UMa system, having an orbital period of 0.5914 days. Different spectral types have been determined by different investigators, but its colour index $B-V=+0.41$ favours a spectral type F3 (Goecking \& Duerbeck 1993). Photometric investigations were published by Knipe (1967), Tapia (1969), Hernandez (1972) and Twigg (1979). The most recent spectroscopic study was made by Goecking \& Duerbeck (1993) who analysed photographic high dispersion spectra by a cross-correlation technique. The spectra with a dispersion of $20 \AA \mathrm{mm}^{-1}$ were obtained on Kodak IIIa-J plates. The useful spectral region ranges from 355 to $475 \mathrm{~nm}$ and the resolution is $0.04 \mathrm{~nm}$. Exposure times varied between 15 and $30 \mathrm{~min}$. The system shows total eclipses, the primary being a transit, the secondary one an occultation. The history of $\epsilon$ Cra up to 1993 was summarized by Goecking \& Duerbeck (1993).
The $V$ light curve of Tapia (1969) and the spectroscopic massratio of Goecking \& Duerbeck (1993) have been used in the present analysis.

\section{Method of analysis and results}

The 1996 version of the Wilson-Devinney DC program and the Binary Maker 2.0 (Bradstreet 1993) have been used for the light curve analysis. The data used are mentioned in Sect. 2, where the description of the individual systems is given. The free parameters were: the phase of conjunction $\phi_{0}$, the inclination $i$, the temperature of the secondary component $T_{2}$, the fractional luminosity of the primary $L_{1}$ and the potential $\Omega$ (according to the mode used), while the fixed (adopted) ones were: the temperature of the primary $T_{1}$ (from the available spectral type), the gravity darkening coefficient $g(0.32$ for convective envelopes, 1.0 for radiative), the albedo A ( 0.5 for convective, 1.0 for radiative), and limb darkening coefficients $x$ (from tables by Claret et al. 1995 and Díaz-Cordovés et al. 1995). The spectroscopic mass ratio $q$ was used as fixed parameter and the third light was zero in all cases. The basic elements derived from the light curve analysis are given in Tables 1 and 2, where all errors given are standard deviations. These elements were combined with the existing spectroscopic data to compute the physical parameters (absolute elements) of the systems, which are given in Table 3 . In the same table the percentage differences between the absolute elements derived from ground based observations and those from Hipparcos observations are also given; the numbers in parentheses are standard deviation errors on the last 
Table 1. Solutions of the light curves from ground based and Hipparcos observations for the near-contact binary stars studied.

\begin{tabular}{l|cc|cc}
\hline \hline Parameter & \multicolumn{2}{|c|}{ RZ Dra (Mode 5) } & \multicolumn{2}{c}{ V1010 Oph (Mode 4) } \\
\hline & $\begin{array}{c}\text { ground based } \\
\text { V observations } \\
\text { (Kreiner et al. 1994) }\end{array}$ & $\begin{array}{c}\text { Hipparcos data } \\
\text { (115 Hp points) }\end{array}$ & $\begin{array}{c}\text { ground based } \\
\text { V observations } \\
\text { (Leung 1974) }\end{array}$ & $\begin{array}{c}\text { Hipparcos data } \\
\text { (78 Hp points) }\end{array}$ \\
\hline$\phi_{0}$ & $0.0000(1)$ & $0.0276(11)$ & $0.0012(2)$ & $0.0012(7)$ \\
$i$ (degrees) & $87.21(27)$ & $84.18(104)$ & $85.86(68)$ & $81.06(95)$ \\
$g_{1}$ & $1.00^{*}$ & $1.00^{*}$ & $1.00^{*}$ & $1.00^{*}$ \\
$g_{2}$ & $0.32^{*}$ & $0.32^{*}$ & $0.32^{*}$ & $0.32^{*}$ \\
$T_{1}(\mathrm{~K})$ & $8150^{*}$ & $8150^{*}$ & $8200^{*}$ & $8200^{*}$ \\
$T_{2}(\mathrm{~K})$ & $4887(30)$ & $5270(125)$ & $5652(33)$ & $5748(73)$ \\
$\Omega_{1}$ & $2.824(4)$ & $2.846(28)$ & $2.7755^{*}$ & $2.7755^{*}$ \\
$\Omega_{2}$ & $2.767^{*}$ & $2.767^{*}$ & $2.7721(280)$ & $2.7772(252)$ \\
$q=m_{2} / m_{1}$ & $0.444(3)$ & $0.444^{*}$ & $0.4485^{*}$ & $0.4485^{*}$ \\
$L_{1} /\left(L_{1}+L_{2}\right)$ & $0.9093(34)$ & $0.9187(148)$ & $0.8964(36)$ & $0.8890(114)$ \\
$x_{1}(V)$ & $0.601^{*}$ & $0.601^{*}$ & $0.598^{*}$ & $0.598^{*}$ \\
$x_{2}(V)$ & $0.783^{*}$ & $0.737^{*}$ & $0.688^{*}$ & $0.680^{*}$ \\
$x_{1}$ (bolo) & $0.603^{*}$ & $0.603^{*}$ & $0.606^{*}$ & $0.606^{*}$ \\
$x_{2}$ (bolo) & $0.581^{*}$ & $0.574^{*}$ & $0.561^{*}$ & $0.557^{*}$ \\
$r_{1}$ (pole) & $0.4145(9)$ & $0.4106(46)$ & $0.4317(18)$ & $0.4356(27)$ \\
$r_{1}$ (side) & $0.4393(12)$ & $0.4342(59)$ & $0.4613(22)$ & $0.4663(36)$ \\
$r_{1}$ (back) & $0.4635(16)$ & $0.4571(73)$ & $0.4909(27)$ & $0.4975(47)$ \\
$r_{2}$ (pole) & $0.2908(4)$ & $0.2905(22)$ & $0.3036(95)$ & $0.3065(56)$ \\
$r_{2}$ (side) & $0.3033(4)$ & $0.3030(22)$ & $0.3189(117)$ & $0.3225(70)$ \\
$r_{2}$ (back) & $0.3359(4)$ & $0.3356(19)$ & $0.3628(219)$ & $0.3693(128)$ \\
$\Sigma\left(\right.$ res) ${ }^{2}$ & 0.0092 & 0.0001 & 0.1924 & 0.0025 \\
\hline As & & & & \\
\hline
\end{tabular}

${ }^{*}$ Assumed.

Table 2. Solutions of the light curves from ground based and Hipparcos observations for the contact binary stars studied.

\begin{tabular}{l|cc|cc}
\hline \hline Parameter & \multicolumn{2}{|c|}{ V566 Oph (Mode 3) } & \multicolumn{2}{c}{$\epsilon$ CrA (Mode 3) } \\
\hline & $\begin{array}{c}\text { ground based } \\
\text { V observations } \\
\text { (Bookmyer 1976) }\end{array}$ & $\begin{array}{c}\text { Hipparcos data } \\
\text { (64 Hp points) }\end{array}$ & $\begin{array}{c}\text { ground based } \\
\text { V observations } \\
\text { (Twigg 1979) }\end{array}$ & $\begin{array}{c}\text { Hipparcos data } \\
\text { (82 Hp points) }\end{array}$ \\
\hline$\phi_{0}$ & $0.0001(1)$ & $0.9524(6)$ & $0.0007(1)$ & $0.0446(12)$ \\
$i$ (degrees) & $80.30(24)$ & $81.92(104)$ & $72.25(62)$ & $73.60(144)$ \\
$g_{1}$ & $0.32^{*}$ & $0.32^{*}$ & $0.32^{*}$ & $0.32^{*}$ \\
$g_{2}$ & $0.32^{*}$ & $0.32^{*}$ & $0.32^{*}$ & $0.32^{*}$ \\
$T_{1}(\mathrm{~K})$ & $7000^{*}$ & $7000^{*}$ & $7100^{*}$ & $7100^{*}$ \\
$T_{2}(\mathrm{~K})$ & $6881(12)$ & $6761(39)$ & $6639(40)$ & $6549(74)$ \\
$\Omega_{1}$ & $2.2575(34)$ & $2.2620(76)$ & $1.9734(160)$ & $1.9828(79)$ \\
$\Omega_{2}$ & $2.2575(34)$ & $2.2620(76)$ & $1.9734(160)$ & $1.9828(79)$ \\
$q=m_{2} / m_{1}$ & $0.2369(12)$ & $0.2369^{*}$ & $0.1120(77)$ & $0.1120^{*}$ \\
$L_{1} /\left(L_{1}+L_{2}\right)$ & $0.7879(25)$ & $0.8018(50)$ & $0.9111(52)$ & $0.9049(74)$ \\
$x_{1}(V)$ & $0.589^{*}$ & $0.589^{*}$ & $0.585^{*}$ & $0.585^{*}$ \\
$x_{2}(V)$ & $0.598^{*}$ & $0.602^{*}$ & $0.608^{*}$ & $0.615^{*}$ \\
$x_{1}$ (bolo) & $0.544^{*}$ & $0.544^{*}$ & $0.545^{*}$ & $0.545^{*}$ \\
$x_{2}$ (bolo) & $0.542^{*}$ & $0.541^{*}$ & $0.541^{*}$ & $0.541^{*}$ \\
$r_{1}$ (pole) & $0.4891(10)$ & $0.4880(18)$ & $0.5352(18)$ & $0.5308(22)$ \\
$r_{1}$ (side) & $0.5342(16)$ & $0.5326(25)$ & $0.5951(22)$ & $0.5909(36)$ \\
$r_{1}$ (back) & $0.5628(21)$ & $0.5609(33)$ & $0.6205(26)$ & $0.6106(40)$ \\
$r_{2}$ (pole) & $0.2608(13)$ & $0.2596(21)$ & $0.2077(16)$ & $0.2005(40)$ \\
$r_{2}$ (side) & $0.2738(16)$ & $0.2723(24)$ & $0.2184(21)$ & $0.2090(31)$ \\
$r_{2}$ (back) & $0.3235(39)$ & $0.3204(50)$ & $0.2498(47)$ & $0.2448(64)$ \\
$\Sigma$ (res) & 0.027 & $2.1 \times 10^{-6}$ & 0.0055 & $3.8 \times 10^{-6}$ \\
& & & & \\
\hline Astme & & & & \\
\hline
\end{tabular}

* Assumed. 

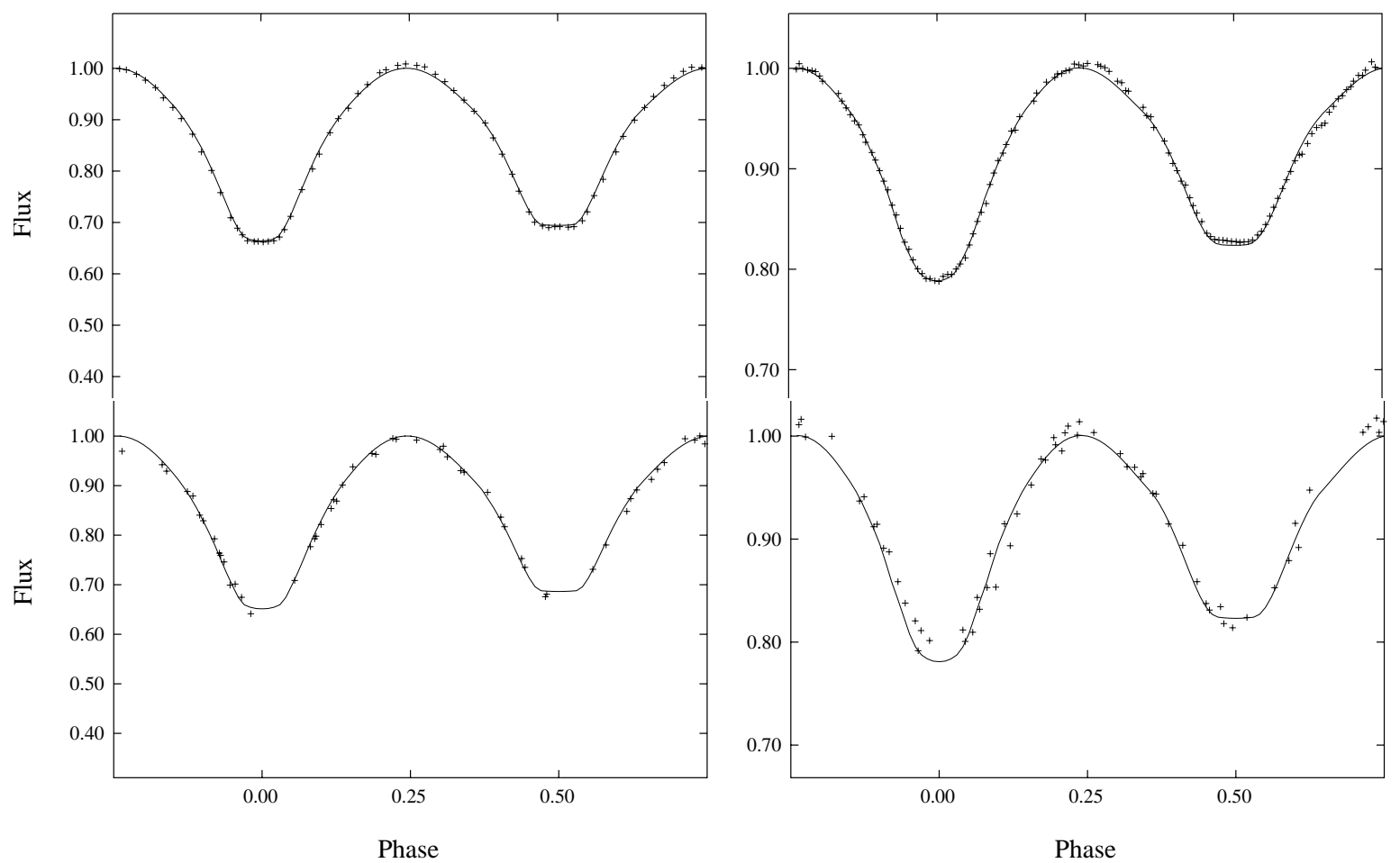

Fig. 2. Light curves of the contact systems V566 Oph (left) and $\epsilon$ CrA (right). The upper curves are from ground based observations and light curve solutions (Boookmyer 1976; Tapia 1969, respectively). The lower curves are from Hipparcos data and our respective light curve solutions (see text for details).

digit. An inspection of the Tables 1 and 2 reveals that a satisfactory agreement exists between the solutions derived from the ground-based and Hipparcos observations. In the case of RZ Dra an extremely good agreement exists between the stellar radii in the two solutions, while a small diference is seen in the inclination and the temperature of the secondary component, as a result of the slightly different depths of the minima. Again, in the case of V1010 Oph, the derived parameters in the two solutions are in very good agreement, except the orbital inclination. This small difference is very probably due to the slightly unequal depths of the primary minimum in the light curves of ground-based and Hipparcos observations, respectively. A small O'Connell effect in the light curve of V1010 Oph has been explained by invoking a cool spot on the cooler secondary component. The derived parameters of V566 Oph in the two solutions are in extremely good agreement, except a very small (a few percent) difference in the inclination and the temperature of the secondary component. Such small differences are expected, since the number of observed points and phase coverage are different in the two cases. For the other contact system, $\epsilon \mathrm{CrA}$, the computed elements are again in very good agreement, despite the fewer points and the poorer phase coverage in the Hipparcos light curves.

\section{Discussion and conclusions}

There are some special advantages of the GAIA mission (with combined astrometric, spectroscopic and photometric observations) compared to the classical ground based approach to eclipsing binary studies. GAIA will provide light curves for millions of faint eclipsing binaries and also the absolute luminosities and temperatures (from the parallaxes and colours). It will then be possible to estimate the absolute stellar radii, the sizes of the orbits via the light curves and then the masses of the systems. Although the expected accuracy will be moderate, the large amount of data will allow us to look for large deviations from the "normal" mass-radius-luminosity relations.

Zwitter (2002, 2003) gives good estimates of the errors on single-star parameters (effective temperature, gravity, metallicity and distance) obtained from a combination of astrometry, photometry and spectroscopy. These error values could be checked by observations of some $4 \times 10^{5}$ eclipsing binaries with $V \leq 15$, of which some $\sim 10^{5}$ will be double-lined. It is expected that for a few tens of thousands of such systems excellent orbital solutions with secure determination of stellar parameters will be obtained. On the other hand, for fainter systems $(V>15)$, although good quality light curves could be obtained, the spectroscopic observations will be of limited accuracy. In these cases only good estimates of stellar parameters can be secured.

In the present study, the computation of the absolute elements of the systems is based on the light curve analysis of photometric observations where the value of mass ratio used is derived from spectroscopic observations with modern techniques. The accuracy of the computed elements depends strongly on both the quality and number of the photometric and spectroscopic observations, and the methods of light curve and spectral analysis. For ground-based observations these methods have reached a very high level, although there is space 
Table 3. Absolute elements in solar units.

\begin{tabular}{lcccccc}
\hline \hline System & $M_{1}$ & $M_{2}$ & $R_{1}$ & $R_{2}$ & $L_{1}$ & $L_{2}$ \\
\hline RZ Dra (1) & $1.40(4)$ & $0.62(3)$ & $1.62(1)$ & $1.12(1)$ & $10.1(4)$ & $1.01(6)$ \\
RZ Dra (2) & $1.42(5)$ & $0.59(4)$ & $1.55(1)$ & $1.11(1)$ & $9.60(47)$ & $0.86(5)$ \\
\% difference & 1.4 & 4.8 & 4.3 & 0.9 & 5 & 15 \\
& & & & & & \\
V1010 Oph (1) & $1.87(12)$ & $0.90(5)$ & $2.08(6)$ & $1.48(8)$ & $10.7(7)$ & $3.47(35)$ \\
V1010 Oph (2) & $1.88(14)$ & $0.89(7)$ & $2.08(1)$ & $1.46(1)$ & $11.7(8)$ & $3.07(8)$ \\
\% difference & 0.5 & 1.1 & 0 & 1.4 & 9.3 & 12 \\
& & & & & & \\
V566 Oph (1) & $1.40(3)$ & $0.33(1)$ & $1.47(1)$ & $0.79(1)$ & $4.57(4)$ & $1.26(1)$ \\
V566 Oph (2) & $1.54(11)$ & $0.36(4)$ & $1.518(3)$ & $0.819(4)$ & $4.99(24)$ & $1.26(2)$ \\
\% difference & 10 & 9.1 & 3.5 & 3.7 & 9.2 & 0.8 \\
& & & & & & \\
$\epsilon$ CrA (1) & $1.75(4)$ & $0.21(2)$ & $2.20(3)$ & $0.80(1)$ & $11.1(1)$ & $1.08(1)$ \\
$\epsilon$ CrA (2) & $1.69(7)$ & $0.22(5)$ & $2.12(12)$ & $0.80(4)$ & $10.3(20)$ & $1.07(14)$ \\
\% difference & 3.4 & 4.8 & 3.6 & 1.2 & 7.2 & 0.9 \\
\hline
\end{tabular}

(1): Ground based observations, (2): GAIA (expected) observations.

for further improvement. For GAIA observations, it was shown by Munari et al. (2001) and Zwitter (2002, 2003), that stellar parameters could be determined (in many cases) at about $2 \%$ accuracy level. Given that GAIA observations will be superior to those of Hipparcos/Tycho mission, the results obtained by using Hipparcos observations should present a lower limit to the GAIA expected accuracy. Another point to be stressed is that for double-lined detached and contact systems the results will be of the accuracy level mentioned before. The problem will be with the near-contact (semi-detached) systems, in which only one spectral line can be observed with medium size ( $\sim 2 \mathrm{~m}$ ) ground-based telescopes. For bright such systems it is possible to observe with large telescopes both lines and disentagle them by modern techniques. The problem will again remain for GAIA spectroscopic observations of semi-detached systems, although the detection limit is increased.

From an inspection of Table 3 it is obvious that the agreement between the fundamental stellar parameters, derived from ground-based and Hipparcos observations is more than satisfactory. Perhaps the most crucial is that, for the near-contact systems, the derived absolute elements from Hipparcos observations differ from those of ground based observations within the limits of the combined errors. For the contact systems, although these differences are larger than in the case of nearcontact systems, they are mostly within the limits of the combined errors. Since GAIA photometry will have excellent precision and much larger number of photometric bands than Hipparcos, it is expected that the resulting stellar parameters will be comparable to those obtained from high quality groundbased observations. This will not be the case for all observed eclipsing binaries, but only for the systems where a good phase coverage (especially during eclipses) is obtained. The eclipse coverage will be better in intermediate passband filters. A good example of the phase coverage of spectroscopic, narrow- and broad-band photometric observations to be obtained by GAIA for a typical double-lined eclipsing system (the GK Dra) is given in Fig. 4 of Zwitter (2003). In the same paper, the accuracies of the fundamental parameters for 6 systems studied so far by Munari et al. (2001) and Zwitter et al. (2003) are given. With some exceptions for individual radii which are not well determined due to the incomplete phase coverage of eclipses, the relative errors in most parameters are $2 \%$ or lower. On the average, errors of the same order were found for the four systems studied in the present work.

The role of GAIA photometry in the study of eclipsing binaries has been reviewed in detail by Munari et al. (2001), Hoeg (2002), Zwitter (2002, 2003) and will not be repeated here. Apart from the fact that GAIA multi-colour photometry will be the only astrophysical information available for most of the brightest one billion stars on the sky, it is worth seeing how the 15 diagnostic bands instead of a single white-light one (GAIA vs. Hipparcos) will impact the accuracy of the light curve solution. It is well-known to light curve modelers that simultaneous fitting by using photometric observations in several bands together with radial velocities can yield consistent results (Kallrath \& Milone 1999). The more the light curves in different wavelegths are used, the more stable the solution is. Also, light curves in different wavelengths are needed for systems with large temperature differences between the two components. On the other hand, the limited number of photometric and spectroscopic observations obtained by GAIA will make it difficult to study intrinsic variability of the binary components. We fully agree with Munari et al. (2001) that, if the same basic set of photometric bands could be used in all the three channels of view, the number of the collected photometric points would proportionally increase with great benefit to the study of variable stars, especially of those exhibiting rapid variations.

Moreover, an estimate of the number of near-contact and contact eclipsing systems, which are expected to be observed by GAIA at its limiting magnitudes, will be given. It has been said elsewhere that GAIA will observe $\sim 4 \times 10^{5}$ eclipsing binaries brighter than $V=15, \sim 10^{5}$ of which will be double-lined 
systems (Munari et al. 2001; Zwitter 2002). If we adopt the evolutionary scenario for W UMa contact systems (Guinan \& Bradstreet 1988), where initially detached binaries with solartype components lose angular momentum via magnetic braking and become contact binaries, we can very roughly estimate the number of contact binaries among the eclipsing binaries observed by GAIA at $V=15$. The resulting number is $\sim 2 \times 10^{5}$, $\sim 5 \times 10^{4}$ of which with double-lined spectra. On the other hand, the General Catalogue of Variable Stars (fourth edition) lists 514 eclipsing systems of W UMa-type with known periods, while about two hundred near-contact systems are known so far (Shaw \& Smith 1999), namely one third of the W UMa-type systems. Therefore, very roughly, GAIA will observe about 4 hundred times more contact and near-contact binaries than those that are known from ground-based observations.

However, a more definite conclusion for the expected GAIA performance on the photometry of eclipsing binaries could be drawn when enough stars will be investigated to cover both various spectral types as well as various kinds of interaction (detached, semi-detached and contact binaries).

Acknowledgements. We would like to thank the anonymous referee for valuable comments and suggestions which significantly improved the content and presentation of this work. I (P.N) am grateful to U. Munari for introducing me to the fascinating world of GAIA. It is also a pleasure to thank U. Munari and T. Zwitter for fruitful discussions on the subject and their help in having access to the very recent relevant bibliography.

\section{References}

Andersen, J. 1991, A\&AR, 3, 91

Bookmyer, B. B. 1976, PASP, 88, 473

Bradstreet, D. H. 1993, BINARY MAKER 2.0 User Manual, Contact Software, Norristown (Pennsylvania)

Ceraski, W. 1907, Astron. Nachr., 174, 265

Corcoran, M. F., Siah, M. J., \& Guinan, E. F. 1991, AJ, 101, 1828

Cousins, A. W. J., \& Cox, A. N. 1950, MNASSA, 9, 90

Cowley, A., Cowley, C., Jaschek, M., \& Jaschek, C. 1969, AJ, 74, 375

Claret, A., Díaz-Cordovés, J., \& Gimenez, A. 1995, A\&AS, 114, 247

Díaz-Cordovés, J., Claret, A., \& Gimenez, A. 1995, A\&AS, 110, 329

Eaton, J. 1986, Acta Astron., 36, 275

Gilmore, G., Perryman, M., Lindegren, L., et al. 1998, Proc. SPIE Conf., 3350, 541

Goecking, K.-D., \& Duerbeck, H. W. 1993, A\&A, 278, 463
Guinan, E., \& Bradstreet, D. H. 1988, in Formation and Evolution of Low Mass Stars, ed. A. K. Dupree, \& M. T. Lago (Dordrecht: Kluwer), 345

Heard, J. F. 1965, J. Roy. Astron. Soc. Canada, 59, 258

Hernández, C. A. 1972, AJ, 77, 152

Hill, G., Fisher, W. A., \& Holmgren, D. 1989, A\&A, 218, 152

Hoeg, E. 2002, in GAIA: A European Project, ed. O. Bienaymé, \& C. Turon, EAS Pub. Ser., 2, 27

Hoffmeister, C. 1935, Astron. Nachr., 255, 401

Kallrath, J., \& Milone, E. F. 1999, Eclipsing Binary Stars (New York: Springer)

Knipe, G. F. G. 1967, Union Obs. Circ., 7, 142

Kreiner, J. M., Pajdosz, G., Tremko, J., \& Zola, S. 1994, A\&A, 285, 459

Lafta, S. J., \& Grainger, J. F. 1985, Ap\&SS, 114, 23

Leung, K.-C. 1974, AJ, 79, 852

Lipari, S. L., \& Sistero, R. F. 1987, AJ, 94, 792

Manimanis, V. N. 2003, Ph.D. dissertation

McLean, B. J. 1983, MNRAS, 204, 817

Munari, U. 1999a, in Proceedings of the ESA Leiden Workshop on GAIA, 23-27 Nov. 1998, Baltic Astron., 8, 73

Munari, U. 1999b, in Proceedings of the ESA Leiden Workshop on GAIA, 23-27 Nov. 1998, Baltic Astron., 8, 123

Munari, U., Tomov, T., Zwitter, T., et al. 2001, A\&A, 378, 477

Munari, U. 2002, in GAIA: A European Project, ed. O. Bienaymé, \& C. Turon, EAS Pub. Ser., 2, 39

Niarchos, P., Rovithis-Livaniou, H., \& Rovithis, P. 1993, Ap\&SS, 203, 197

Pace, O. 2003, in GAIA Spectroscopy, Science and Technolog, ed. U. Munari, ASP Conf. Ser., 298, 13

Perryman, M., De Boer, K. S., Gilmore, G., et al. 2001, A\&A, 369, 339

Popper, D. M. 1966, AJ, 71, 175

Rucinski, S. M., Lu, W., \& Mochnacki, S. W. 2000, AJ, 120, 1133

Shaw, J. S. 1990, in Active Close Binaries, ed. C. Ibanoglu (Dordrecht: Kluwer), 241

Shaw, J. S., Guinan, E. F., \& Garasi, G. J. 1990, BAAS, 22, 1296

Shaw, J. S., \& Smith, E. C. 1999, BAAS, 31, 1486

Strohmeier, W., Knigge, R., \& Ott, H. 1964, IBVS No. 74

Struve, O. 1946, ApJ, 103, 76

Tapia, S. 1969, AJ, 74, 533

Twigg, L. W. 1979, MNRAS, 189, 907

van Hamme, W., \& Wilson, R. E. 1985, A\&A, 152, 25

Zwitter, T. 2002, in Exotic Stars as Challenges to Evolution, ed. C. A. Toot, \& W. van Hamme, ASP Conf. Ser., 279, 31

Zwitter, T. 2003, in GAIA Spectroscopy, Science and Technology, ed. U. Munari, ASP Conf. Ser., 298, 329 